\title{
Extension of geodesic algebras to continuous genus
}

\author{
L. Chekhov,${ }^{*}$ J. E. Nelson,$^{\dagger}$ and T. Regge ${ }^{\ddagger}$
}

\begin{abstract}
Using the Penner-Fock parameterization for Teichmüller spaces of Riemann surfaces with holes, we construct the string-like free-field representation of the Poisson and quantum algebras of geodesic functions in the continuous-genus limit. The mapping class group acts naturally in the obtained representation.
\end{abstract}

Mathematics Subject Classification (2000) 14D21, 46L87, 53C22.

Keywords: 3D gravity, moduli spaces, geodesics, large-genus limit

\section{Introduction}

The classical phase space of Einstein gravity on a 3D manifold is the Teichmüller space of its boundary [1]. This Teichmüller space has a canonical (Weil-Petersson) Poisson structure with the mapping class group as its symmetry group. When quantizing this structure [2, 3], the algebra of observables in the corresponding quantum theory is the noncommutative deformation

*Steklov Mathematical Institute, Gubkina 8, 117966, GSP-1, Moscow, Russia; Institute for Experimental and Theoretical Physics, Moscow, Russia; Laboratoire Internationale Franco-Russie. Email: chekhov@mi.ras.ru.

${ }^{\dagger}$ Dipartimento di Fisica Teorica, Università degli Studi di Torino and Istituto Nazionale di Fisica Nucleare, Sezione di Torino, via Pietro Giuria 1, 10125, Torino, Italy, email: nelson@to.infn.it.

${ }^{\ddagger}$ Dipartimento di Fisica, Politecnico di Torino, Corso Duca degli Abruzzi, 10129 Torino, Italy. 
of the *-algebra of functions whose Poisson brackets become commutation relations with quantum parameter $\hbar$. The symmetry group acts on the algebra of observables by automorphisms generated by unitary operators of the quantum mapping class group. These observables are the lengths of closed geodesics on a Riemann surface, and they were constructed from the Teichmüller space coordinates using the graph technique of Penner 4 and Fock [5]. The induced geodesic algebras with Goldman Poisson brackets [6] are exactly those obtained in the first-order formalism description of $2+1$ dimensional gravity by Nelson, Regge, and Zertuche [7, 8].

In Section 2, we briefly review the first-order formalism of 3D (i.e. $2+1$ ) gravity in which Goldman brackets appear dynamically; in Section 3, we review the graph description of Teichmüller spaces of Riemann surfaces with holes and introduce a set of geodesic functions, the observables in the theory. In Section 4, we consider the specific basis of observables for which the Nelson-Regge Poisson algebra is satisfied. In Section 5, using the PennerFock coordinates, we construct the limiting expressions for geodesic functions in the limit of infinite, or continuous genus and find that they satisfy a simple string-like (or CFT-like) representation in terms of free fields. The Poisson relations for these fields coincide with those of the string coordinates. We also consider the action of the subclass of the mapping class group transformations generated by Dehn twists along geodesics from those considered by Nelson and Regge 8, and show that these symmetries act naturally within the above free-field representation. We construct the quantization of the Poisson geodesic algebras in the limit of infinite genus in Section 6.

\section{The first-order formalism in 3D gravity}

Here we briefly describe the approach to $2+1$-dimensional gravity based on the first-order formalism [7, 8]. For the standard Einstein-Hilbert action (without matter) and cosmological constant $\Lambda$

$$
I=\frac{1}{16 \pi G} \int_{M} \mathrm{~d}^{3} x \sqrt{-g}(R-2 \Lambda),
$$

the classical solutions are constant curvature spaces with topology $M \sim$ $[0,1] \times S$, where $S$ is a closed $2 \mathrm{D}$ surface.

In the first-order formalism, the fundamental independent variables are a spin connection $w_{\mu}^{a b}$, and a local frame (or "dreibein") $e_{\mu}^{a}$ satisfying 
$\eta_{a b} e_{\mu}{ }^{a} e_{\nu}^{b}=g_{\mu \nu}, \eta_{a b}=\operatorname{diag}\{-++\}$. Introducing the one-forms $e^{a}=e_{\mu}{ }^{a} \mathrm{~d} x^{\mu}$ and $w^{a}=\frac{1}{2} \epsilon^{a b c} w_{\mu b c} \mathrm{~d} x^{\mu}$ the action (2.1) becomes

$$
I=-2 \int_{M}\left(e \wedge d w+\frac{1}{2} e \wedge w \wedge w+\frac{\Lambda}{6} e \wedge e \wedge e\right) .
$$

Setting $\Lambda=-1 / \ell^{2}<0$, and introducing the real variables $A^{( \pm) a}=w^{a} \pm \frac{1}{\ell} e^{a}$, we obtain the Chern-Simons (CS) theory with an $S O(2,1) \times S O(2,1)$ gauge potential

$$
I\left[A^{(+)}, A^{(-)}\right]=I_{C S}\left[A^{(+)}\right]-I_{C S}\left[A^{(-)}\right],
$$

where

$$
I_{C S}[A]=\frac{k}{4 \pi} \int_{M} \operatorname{tr}\left(A \wedge d A+\frac{1}{3} A \wedge A \wedge A\right)
$$

is the standard CS action.

The two dynamical (spacelike) components satisfy the Poisson brackets

$$
\left\{A_{i}^{( \pm) a}(x), A_{j}^{( \pm) b}\left(x^{\prime}\right)\right\}= \pm \frac{1}{\ell} \epsilon_{i j} \eta^{a b} \delta^{2}\left(x-x^{\prime}\right),
$$

while all of the $A^{+}$variables Poisson commute with all of the $A^{-}$variables. The holonomies, or geodesic functions, are

$$
G_{\gamma}^{( \pm)}=P \exp \left\{\int_{\gamma} A_{i}^{( \pm) a} T_{a} \mathrm{~d} x^{i}\right\}
$$

and their Poisson brackets were first found by Goldman [6]. However, these brackets and the skein relations can be obtained from different considerations based on the graph description.

\section{Classical Teichmüller spaces. The graph technique}

Classically, Teichmüller spaces $\mathcal{T}_{g, s}$ of Riemann surfaces of genus $g$ with $s$ holes $(s>0)$ are spaces of complex structures on a (possibly open) Riemann surface $S$ modulo diffeomorphisms homotopy equivalent to the identity. In the vicinity of a boundary component, the complex structure is isomorphic as a complex manifold either to an annulus (hole) or to a punctured disc (puncture). 
An oriented 2D surface can be continuously conformally transformed to a constant curvature surface. The Poincaré uniformization theorem states that any complex surface $S$ of a constant negative curvature is a quotient of the upper half-plane $\mathbb{H}_{+}$endowed with the hyperbolic metric $d s^{2}=d z d \bar{z} /(\Im z)^{2}$ with respect to (w.r.t.) the action of a discrete Fuchsian subgroup $\Delta(S)$ of the automorphism group $P S L(2, \mathbb{R})$,

$$
S=\mathbb{H}_{+} / \Delta(S)
$$

Any hyperbolic homotopy class of closed curves $\gamma$ contains a unique closed geodesic of the length $l(\gamma)=\log \left|\lambda_{1} / \lambda_{2}\right|$, where $\lambda_{1}$ and $\lambda_{2}$ are (different) real eigenvalues of the element of $\operatorname{PSL}(2, \mathbb{R})$ corresponding to $\gamma$. These lengths are the desired physical observables.

The central point of the construction is the description of the Teichmüller space $\mathcal{T}_{g, s}(S)$ in terms of fat graphs [5], which we briefly describe.

Let a fat graph $\Gamma$ be a graph with a given cyclic ordering of the edges entering each vertex. We take all vertices to be three-valent, and coordinatize the Teichmüller space by associating real numbers $Z_{\alpha} \in \mathbb{R}$ to unoriented edges. In this way we obtain the set $\left\{Z_{\alpha} \mid \alpha \in E(\Gamma)\right\}$, where $E(\Gamma)$ is the set of all (unoriented) edges of the graph $\Gamma$. We also let $V(\Gamma)$ denote the set of vertices of a graph $\Gamma$.

A fat graph that is embedded into an oriented surface inherits the canonical fat structure from the surface orientation. Denote by $\Gamma(S)$ the set of all isotopy classes of three-valent fat graphs embedded in the surface $S$ in such a way that the surface is shrinkable to the image. For any $u \in \Gamma(S)$ and for any given edge of $u$, associate a standard morphism $u \mapsto v$, where $v \in \Gamma(S)$ is obtained from $u$ by a flip of the edge (shown in Figure 1 below). These morphisms, together with symmetries of a graph, generate the mapping class group.

A three-valent graph $\Gamma$ of genus $g$ with $s$ holes therefore generates an isomorphism between the set of points of $\mathcal{T}_{g, s}(S)$ and the set $\mathbb{R}^{\# \text { edges }}$ of edges of this graph endowed with real numbers [5].

In order to parameterize closed geodesics (paths over edges of the graph) on the Riemann surface, associate the matrix $X_{Z_{\alpha}} \in P S L(2, \mathbb{R})$ corresponding to a Möbius transformation to each edge $\alpha$,

$$
X_{Z_{\alpha}}=\left(\begin{array}{cc}
0 & -\mathrm{e}^{Z_{\alpha} / 2} \\
\mathrm{e}^{-Z_{\alpha} / 2} & 0
\end{array}\right)
$$


and introduce the matrices for "right" and "left" turns

$$
R=\left(\begin{array}{rr}
1 & 1 \\
-1 & 0
\end{array}\right), \quad L \equiv R^{2}=\left(\begin{array}{rr}
0 & 1 \\
-1 & -1
\end{array}\right)
$$

Let a closed path in the graph $\Gamma$ be any oriented path (no turning back), which starts and terminates at the same edge of the graph. The product of matrices standing on consecutive edges and turns of the path is

$$
P_{Z_{1} \ldots Z_{k}}=L X_{Z_{k}} L X_{Z_{k-1}} R X_{Z_{k-2}} \ldots R X_{Z_{2}} L X_{Z_{1}}
$$

In this way one obtains [5] a one-to-one correspondence between the set of conjugacy classes of oriented paths $\left\{P_{Z_{1} \ldots Z_{k}}\right\}$ and closed (oriented) geodesics $\{\gamma\}$ on the surface $S$. The length $l(\gamma)$ of a geodesic is determined from

$$
G_{\gamma} \equiv 2 \cosh (l(\gamma) / 2)=\operatorname{tr} P_{Z_{1} \ldots Z_{k}}
$$

We shall call $G_{\gamma}$ a geodesic function. It should be identified with the geodesic function of equation (2.6) when $\Lambda=-1$.

In the $\left\{Z_{\alpha}\right\}$ coordinates a canonical Poisson structure (the Weil-Petersson structure) $B_{\mathrm{wP}}$ on $\mathcal{T}_{g, s}(S)$ is

$$
B_{\mathrm{wP}}=\sum_{v \in V(\Gamma)} \sum_{i=1}^{3} \frac{\partial}{\partial Z_{v_{i}}} \wedge \frac{\partial}{\partial Z_{v_{i+1}}}
$$

where $v_{i}, i=1,2,3 \bmod 3$, label the cyclically ordered edges incident on a vertex $v$. This structure is degenerate, and its Casimir functions are exactly the lengths of the geodesics encircling holes.

The graph transformation that preserves the Poisson structure (3.5) under the flip operation and satisfies the pentagon identity [2] is shown in Figure 1 where

$$
\{A, B, C, D, Z\} \rightarrow\{A+\phi(Z), B-\phi(-Z), C+\phi(Z), D-\phi(-Z),-Z\}
$$

and classically $\phi(Z)=\log \left(\mathrm{e}^{Z}+1\right)[\underline{5}$.

It was shown in 2 that the transformation (3.6) preserves the traces of products over paths (3.4), i.e. the classical geodesic lengths are invariant under the action of the mapping class group. 


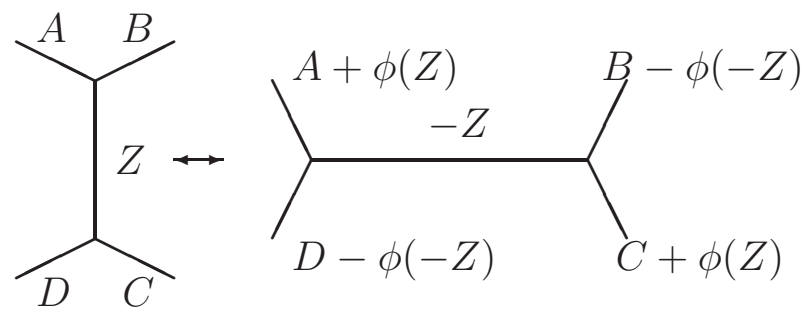

Figure 1

The functions $G_{\gamma}$ (3.4) studied in [6] satisfy a multiplicative Poisson bracket algebra over $\mathbb{Z}$.

The classical skein relation follows from the formula $\operatorname{tr}(A B)+\operatorname{tr}\left(A B^{-1}\right)-$ $\operatorname{tr} A \cdot \operatorname{tr} B=0$, which holds for arbitrary $2 \times 2$ matrices $A$ and $B$ with unit determinants. This can be represented graphically as

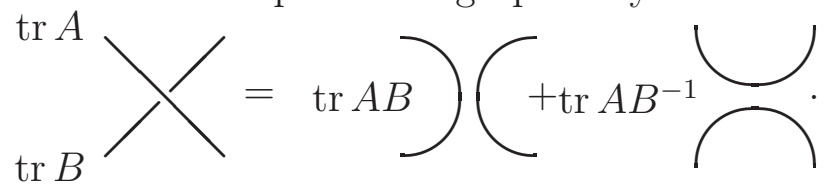

Using this relation we can disentangle any set of crossing geodesics; the basis elements are therefore geodesic laminations (GLs), namely sets of nonintersecting and nonselfintersecting geodesics on $S$.

Consider now the Poisson structure of geodesic functions. Two nonintersecting geodesics have a trivial bracket. Because a Poisson bracket $\left\{G_{1}, G_{2}\right\}$ of two geodesic functions satisfies the Leibnitz rule, it suffices to consider only a single intersection of two geodesics. Graphically, we obtain the relation

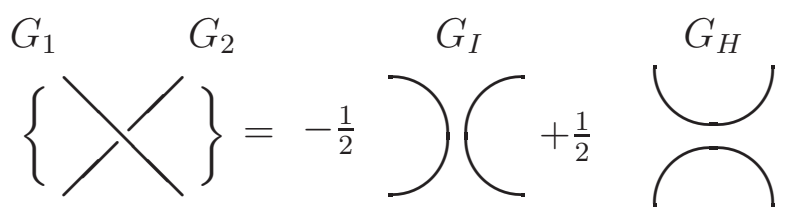

which was obtained in [6] in the continuous parameterization.

A geodesic is said to be graph simple if it passes through each edge of a graph no more than once. The set of graph simple geodesics is obviously finite and depends on the choice of the combinatorial type of a representing graph. These sets, although obviously noninvariant under the mapping class group action, form a convenient basis for closed finite-dimensional geodesic algebras.

Example 1 For the torus $\left(\mathcal{T}_{1,1}\right)$, there are three generators $X, Y, Z$ satisfying the Poisson brackets

$$
\{X, Y\}=\{Y, Z\}=\{Z, X\}=2
$$


and with Casimir $X+Y+Z$. Correspondingly there are only three graph simple geodesics whose geodesic functions are

$$
\begin{aligned}
& G_{X}=\operatorname{tr} L X_{Y} R X_{Z}=\mathrm{e}^{-Y / 2-Z / 2}+\mathrm{e}^{-Y / 2+Z / 2}+\mathrm{e}^{Y / 2+Z / 2}, \\
& G_{Y}=\operatorname{tr} L X_{Z} R X_{X}=\mathrm{e}^{-Z / 2-X / 2}+\mathrm{e}^{-Z / 2+X / 2}+\mathrm{e}^{Z / 2+X / 2}, \\
& G_{Z}=\operatorname{tr} L X_{X} R X_{Y}=\mathrm{e}^{-X / 2-Y / 2}+\mathrm{e}^{-X / 2+Y / 2}+\mathrm{e}^{X / 2+Y / 2} .
\end{aligned}
$$

Introducing the geodesic function $\widetilde{G}_{Z}=\operatorname{tr} R X_{Z} R X_{X} L X_{Z} L X_{Y}$ obtained from $G_{Z}$ by the flip transformation (3.6), it follows that $\left\{G_{X}, G_{Y}\right\}=\widetilde{G}_{Z} / 2-$ $G_{Z} / 2$, and since relation (3.7) implies that $G_{X} G_{Y}=G_{Z}+\widetilde{G}_{Z}$, we obtain

$$
\left\{G_{X}, G_{Y}\right\}=\frac{1}{2} G_{X} G_{Y}-G_{Z}
$$

i.e., the classical Poisson algebra which closes on the subset $\left\{G_{X}, G_{Y}, G_{Z}\right\}$ of geodesic functions (other relations are obtained from cyclic permutations in (3.10) ) at the price of introducing elements of the second order in the r.h.s.

\section{Poisson algebras of geodesics}

In order to generalize Example 1 we must find a graph on which graph simple geodesics constitute a convenient algebraic basis. This is shown in Figure 2 where $m$ edges crosswise connect two horizontal line subgraphs; note that the apparent vertices at the corners of these subgraphs are not vertices. In this picture graph simple closed geodesics are those and only those that pass along exactly two different "vertical" edges of the graph; they are therefore enumerated by the numbers of these edges, and we denote them by $\mathcal{G}_{i j}$ where $i<j$. The geodesic Poisson algebra for $\mathcal{G}_{i j}$ is

$$
\left\{\mathcal{G}_{i j}, \mathcal{G}_{k l}\right\}=\left\{\begin{array}{l}
0, \quad j<k, \\
0, \quad k<i, j<l, \\
\mathcal{G}_{i k} \mathcal{G}_{j l}-\mathcal{G}_{k j} \mathcal{G}_{i l}, \quad i<k<j<l \\
\frac{1}{2} \mathcal{G}_{i j} \mathcal{G}_{j l}-\mathcal{G}_{i l}, \quad j=k, \\
\mathcal{G}_{j l}-\frac{1}{2} \mathcal{G}_{i j} \mathcal{G}_{i l}, \quad i=k, j<l \\
\mathcal{G}_{i k}-\frac{1}{2} \mathcal{G}_{i j} \mathcal{G}_{k j}, \quad j=l, i<k .
\end{array}\right.
$$

This algebra (4.1) is exactly that found by Nelson and Regge 9].

The graph in Figure 2 has genus $\frac{m}{2}-1$ and two holes if $m$ is even and genus $(m-1) / 2$ and one hole if $m$ is odd. Such geodesic bases for $m$ even 
and smooth Riemann surfaces were considered in [7]. The Poisson algebras of geodesics obtained there coincide exactly with (4.1). These are the $s o_{q}(m)$ algebras whose representations were constructed in [10].

In the mathematical literature, the algebra (4.1) has also appeared as the Poisson algebra of monodromy data (Stokes matrices) of some matrix differential equation [11 and as the symplectic groupoid of upper-triangular matrices $A$ [12]. For $m \times m$-matrices, in general we have $\left[\frac{m}{2}\right]$ central elements generated by the polynomial invariants $f_{\mathcal{G}}(\lambda) \equiv \operatorname{det}\left(\mathcal{G}+\lambda \mathcal{G}^{T}\right)=\sum f_{i}(\mathcal{G}) \lambda^{i}$. The total Poisson dimension $d$ of the algebra (4.1) is $\frac{m(m-1)}{2}-\left[\frac{m}{2}\right]$, and for $m=3,4,5,6, \ldots$ we have $d=2,4,8,12, \ldots$. The dimensions of the corresponding Teichmüller spaces are $D=2,4,8,10, \ldots$, so that the Teichmüller spaces are embedded as the Poisson leaves into the algebra (4.1). Starting from the genus 2 surface with two holes, the dimensions of these special leaves become smaller than the highest dimensions of the Poisson representations of (4.1).

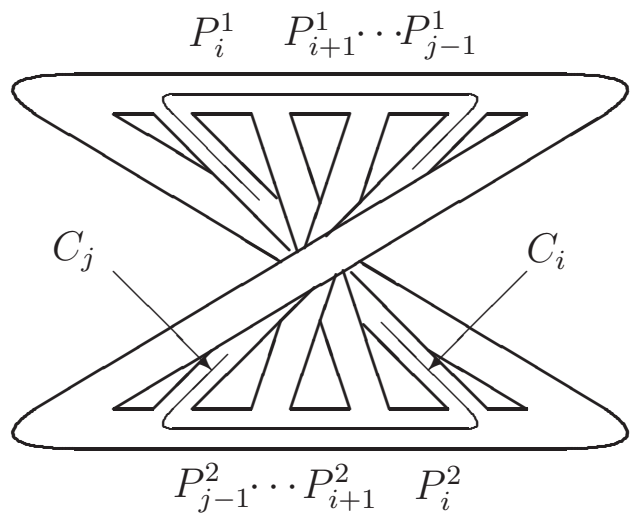

Figure 2

\section{The free-field limit of Nelson-Regge alge- bras}

To produce an infinite-genus limit of geodesic algebras we consider graphs as in Figure 2 with the number of "rungs" of the crossed ladder tending to infinity and replace the index $i$ by a continuous variable $x$. With the parameterization (3.3), we introduce three fields $P^{1,2}(x)$ and $C(x)$ corresponding to the respective quantities living on upper horizontal, lower horizontal, and 
vertical edges. The form of the Poisson relations we wish to obtain can be easily read off from equation (4.1). To be precise, let $x_{1}<x_{2}<x_{3}<x_{4}$. Then,

$$
\left\{\mathcal{G}_{x_{1}, x_{3}}, \mathcal{G}_{x_{2}, x_{4}}\right\}=\mathcal{G}_{x_{1}, x_{2}} \mathcal{G}_{x_{3}, x_{4}}-\mathcal{G}_{x_{1}, x_{4}} \mathcal{G}_{x_{2}, x_{3}},
$$

where all quantities in the products on the r.h.s. mutually commute. In this limit, we disregard all the boundary cases where some of the points $x_{i}$, $i=1, \ldots, 4$, would coincide.

It is straightforward to calculate the geodesic function corresponding to the path indicated in Figure 2 with (still discrete) variables $P_{k}^{1,2}$ and $C_{k}$. We obtain

$$
\begin{aligned}
& \operatorname{tr} L X_{P_{i}^{2}} R \cdots R X_{P_{j-2}^{2}} R X_{P_{j-1}^{2}} L X_{C_{j}} R X_{P_{j-1}^{1}} L \cdots X_{P_{i+1}^{1}} L X_{P_{i}^{1}} R X_{C_{i}} \\
= & 4 s_{1} s_{2} \cosh \left(C_{j} / 2\right) \cosh \left(C_{i} / 2\right)+2 \mathrm{e}^{C_{i} / 2} \cosh \left(C_{j} / 2\right)\left(\mathrm{e}^{\Sigma^{1}+\Sigma^{2}}+s_{1} \mathrm{e}^{\Sigma^{2}}+s_{2} \mathrm{e}^{\Sigma^{1}}\right) \\
+ & 2 \mathrm{e}^{-C_{j} / 2} \cosh \left(C_{i} / 2\right)\left(\mathrm{e}^{-\Sigma^{1}-\Sigma^{2}}+s_{1} \mathrm{e}^{-\Sigma^{2}}+s_{2} \mathrm{e}^{-\Sigma^{1}}\right) \\
+ & 2 \mathrm{e}^{C_{i} / 2-C_{j} / 2} \cosh \left(\Sigma^{1}-\Sigma^{2}\right),
\end{aligned}
$$

where $\Sigma^{1,2} \equiv \sum_{k=i}^{j-1} P_{k}^{1,2}$ and

$$
s_{1}=\sum_{k=i+1}^{j-1} \mathrm{e}^{-\sum_{l=i}^{k-1} P_{l}^{1} / 2+\sum_{l=k}^{j-1} P_{l}^{1} / 2}, \quad s_{2}=\sum_{k=i}^{j-2} \mathrm{e}^{-\sum_{l=i}^{k} P_{l}^{2} / 2+\sum_{l=k+1}^{j-1} P_{l}^{2} / 2} .
$$

In the limit of an infinite number of vertical lines, typical differences $|i-j|$ become infinitely large, and in order to manage it we introduce a scaling parameter $\Delta$ that ensures that the combinations $\Delta|i-j| \equiv\left|x_{i}-x_{j}\right|$ remain finite. To obtain continuously parameterized expressions from (5.2), we simultaneously rescale $\mathcal{G}_{x_{i}, x_{j}}=\Delta^{2} \cdot \mathcal{G}_{i j}$ in order to replace summations by integrations in (5.3). Simultaneously, we replace summations by integrations over a continuous variable in the exponentials in the same formula. ${ }^{1}$ The algebra (5.1) is obviously invariant under such a rescaling, since this reduces to the global additive renormalization of all geodesic lengths $l(\gamma)$. In this field limit the expression (5.2) becomes drastically simplified, in fact only the first term containing the product $s_{1} s_{2}$ survives, and we obtain

$$
\mathcal{G}_{x_{i} x_{j}}=\int_{x_{i}}^{x_{j}} d y \int_{x_{i}}^{x_{j}} d z 4 \cosh \left(C\left(x_{i}\right) / 2\right) \cosh \left(C\left(x_{j}\right) / 2\right) \times
$$

\footnotetext{
${ }^{1}$ Classically, this corresponds to a situation where we also scale $P_{i}^{1,2} \rightarrow P_{i}^{1,2} / \Delta$, i.e., we work in the domain of small variables $P_{i}$. However, we do not scale the Poisson (and commutation) relations for these variables.
} 


$$
\times \exp \left\{\int_{x_{i}}^{x_{j}} d \xi \epsilon(y-\xi) P^{1}(\xi) d \xi / 2+\int_{x_{i}}^{x_{j}} d \rho \epsilon(z-\rho) P^{2}(\rho) d \rho / 2\right\}(5
$$

where $\epsilon(x)=\{+1, x>0 ;-1, x<0\}$.

In this field limit, the field $C(x)$ can be scaled in two possible ways. First, it becomes a single point when integrated over the variable $x$. That is, if $C(x)$ remains quantum it cannot contribute to the total expression (5.4) since now the fields $P^{1,2}(\xi)$ are macroscopic ensembles of the quantum fields $P_{k}^{1,2}$. An alternative is to fix $C(x)$ to be a macroscopic classical field. (This should be related to a deformation of the initial Riemann surface in the continuous-genus limit; we will discuss it elsewhere.) In this case, the fields $C(x)$ becomes commutative. This also reflects the fact that in the continuous limit the algebra (5.1) becomes conformally invariant. The only nontrivial commutation relations are now

$$
\left\{P^{1,2}(x), P^{1,2}(y)\right\}=\delta^{\prime}(x-y), \quad\left\{P^{1}(x), P^{2}(y)\right\} \equiv 0,
$$

i.e. the relations for string coordinates $\partial X(\sigma, \tau)$ at coincident proper times $\tau$ (the free-field representation). That is, if the variables $x, y$ lie in the interval $[0,2 \pi]$, then by representing the $P^{1,2}(x)$ as

$$
P^{1,2}(x)=\frac{1}{\sqrt{2 \pi}} \sum_{l=-\infty}^{+\infty} \mathrm{e}^{i l x} \alpha_{l}^{1,2}
$$

we find the standard CFT Poisson relations $\left\{\alpha_{l}^{\mu}, \alpha_{p}^{\nu}\right\}=l \delta_{l+p} \delta^{\mu \nu}, \mu, \nu=1,2$.

It is convenient to introduce the integrated variables $X^{i}(x)$ where $P^{i}(x)=$ $\partial X^{i}(x)$. Their Poisson relations become

$$
\left\{X^{1,2}(x), X^{1,2}(y)\right\}=\epsilon(x-y) / 2, \quad\left\{X^{1}(x), X^{2}(y)\right\} \equiv 0,
$$

and then the quantities $\mathcal{G}_{x_{i} x_{j}}$ become integrals of local field insertions:

$$
\begin{aligned}
\mathcal{G}_{x_{i} x_{j}}= & \int_{x_{i}}^{x_{j}} d y \int_{x_{i}}^{x_{j}} d z 4 \cosh \left(C\left(x_{i}\right) / 2\right) \cosh \left(C\left(x_{j}\right) / 2\right) \times \\
& \times \mathrm{e}^{X^{1}\left(x_{i}\right) / 2+X^{1}\left(x_{j}\right) / 2+X^{2}\left(x_{i}\right) / 2+X^{2}\left(x_{j}\right) / 2-X^{1}(y)-X^{2}(z)},
\end{aligned}
$$

It can be checked that the Poisson relations (5.1) follow from (5.7) and (5.6).

We now consider the boundary conditions for the free-field variables $P^{1,2}(\xi)$. Consider a variation of Figure 2, where the lower part of the picture 
is inverted i.e. twisted and opened out, so that all rungs are twisted, and become vertical strips connecting the upper and lower parts of the graph. The variables $P^{1,2}(\xi)$ live on these strips. We briefly return to the discrete variables, $P_{i}^{1,2}, i=1, \ldots, n, n=m-3$, and let the variables on the two corners (twisted rungs) be $P_{n+1}^{1}$ and $P_{0}^{2}$. It is easy to see that the only nonzero Poisson brackets for the $P_{i}^{1,2}$ are

$$
\left\{P_{i}^{1}, P_{i+1}^{1}\right\}=\left\{P_{i-1}^{2}, P_{i}^{2}\right\}=1, \quad i=1, \ldots, n
$$

and

$$
\left\{P_{n+1}^{1}, P_{n}^{2}\right\}=\left\{P_{n+1}^{1}, P_{n}^{1}\right\}=\left\{P_{1}^{1}, P_{0}^{2}\right\}=\left\{P_{1}^{2}, P_{0}^{2}\right\}=-1 .
$$

That is, the variables $P^{1}(\xi)$ and $P^{2}(\xi)$ exhibit a mirror-like symmetry.

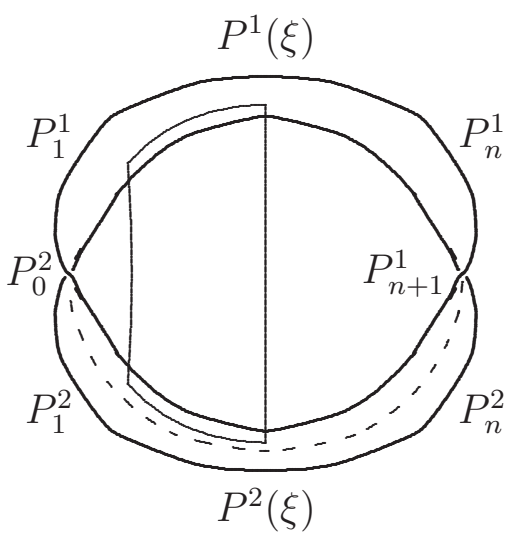

Figure 3

For simplicity, we discard in Figure 3 all the $C$-variable rungs of the diagram (all of them are vertical twisted lines joining symmetrical points $\xi$ on the upper and lower arcs on which the variables $X^{1}(\xi)$ and $X^{2}(\xi)$ reside). We keep only two such lines with variables $P_{0}^{2}$ and $P_{n+1}^{1}$ in order to exhibit the possible boundary conditions. It seems plausible that the boundary relations (5.9) imply Neumann type boundary conditions for the string-like variables $X^{1,2}(\sigma)$ :

$$
P^{1}(0) \equiv \partial X^{1}(0)=P^{2}(0) \equiv \partial X^{2}(0)
$$

and

$$
P^{1}(2 \pi) \equiv \partial X^{1}(2 \pi)=P^{2}(2 \pi) \equiv \partial X^{2}(2 \pi) .
$$

In the next section, we consider the quantization of the functions (5.7) and construct their quantum algebra. 


\section{Quantization}

Let $\mathcal{T}_{g, n}^{\hbar}(\Gamma)$, where $\Gamma \in \Gamma(S)$, be the algebra generated by the generators $Z_{\alpha}^{\hbar}$ (one generator per one (unoriented) edge $\alpha$ ) with the relations

$$
\left[Z_{\alpha}^{\hbar}, Z_{\beta}^{\hbar}\right]=2 \pi i \hbar\left\{Z_{\alpha}, Z_{\beta}\right\}
$$

(cf. (3.5)) with the $*$-structure $\left(Z_{\alpha}^{\hbar}\right)^{*}=Z_{\alpha}^{\hbar}$. Here $Z_{\alpha}$ and $\{\cdot, \cdot\}$ stand for the respective coordinate function and the Poisson bracket on the classical Teichmüller space.

To any flip morphism, we now associate a homomorphism (Figure 1) of the corresponding $*$-algebras that is idempotent and satisfies the five-term relation [2]. This morphism is given by (3.6) with the (quantum) function 13. 14.

$$
\phi(z) \equiv \phi^{\hbar}(z)=-\frac{\pi \hbar}{2} \int_{\Omega} \frac{\mathrm{e}^{-i p z}}{\sinh (\pi p) \sinh (\pi \hbar p)} \mathrm{d} p,
$$

where the contour $\Omega$ goes along the real axis bypassing the origin from above.

For any $\gamma$, the quantum geodesic operator $G_{\gamma}^{\hbar}$ is

$$
G_{\gamma}^{\hbar} \equiv \underset{\times}{\times} \operatorname{tr} P_{Z_{1} \ldots Z_{n} \times} \equiv \sum_{\substack{j \in J \\ \kappa \in\{j\}}} \exp \left\{\frac{1}{2} \sum_{\alpha \in E(\Gamma)}\left(m_{j}(\gamma, \alpha) Z_{\alpha}^{\hbar}+2 \pi i \hbar c_{j}^{\kappa}(\gamma, \alpha)\right)\right\},
$$

where the quantum ordering $\underset{\times}{\times} \cdot \times$ implies that we must change the classical expression by introducing additional integer coefficients $c_{j}^{\kappa}(\gamma, \alpha)$ determined from the following conditions.

1. The mapping class group action $\Delta(S)$ (3.6) with $\phi^{\hbar}(z)$ from (6.2) preserves the set $\left\{G_{\gamma}^{\hbar}\right\}$, i.e., for any $\delta \in \Delta(S)$ and any closed path $\gamma$, we have $\delta\left(G_{\gamma}^{\hbar}\right)=G_{\delta \gamma}^{\hbar}$.

2. Geodesic algebra. The product of two quantum geodesics is a linear combination of quantum geodesic laminations (QGLs) governed by the quantum skein relation [16] below. In analogy with the classical case, a QGL is a set of self- and mutually nonintersecting quantum geodesics.

3. Unorientedness. Quantum traces of direct and inverse geodesic operators coincide.

4. If the closed paths $\gamma$ and $\gamma^{\prime}$ do not intersect, then the operators $G_{\gamma}^{\hbar}$ and $G_{\gamma^{\prime}}^{\hbar}$ commute. Therefore, the order of quantum geodesic functions entering a QGL is irrelevant. 
We introduce the Weyl ordering $: \mathrm{e}^{a_{1}} \mathrm{e}^{a_{2}} \cdots \mathrm{e}^{a_{n}}$ : $\equiv \mathrm{e}^{a_{1}+\cdots+a_{n}}$ for any set $\left\{a_{i}: a_{i} \neq-a_{j}\right\}$. It was found in [15] that for a graph simple geodesic, the quantum ordering is the Weyl ordering.

If $G_{1}^{\hbar}$ and $G_{2}^{\hbar}$ are two non selfintersecting quantum geodesics with one nontrivial intersection, then

$$
G_{1}^{\hbar} G_{2}^{\hbar}=\mathrm{e}^{-i \pi \hbar / 2} G_{Z}^{\hbar}+\mathrm{e}^{i \pi \hbar / 2} \widetilde{G}_{Z}^{\hbar}
$$

That is, graphically, we obtain the Turaev [16] quantum skein relation,

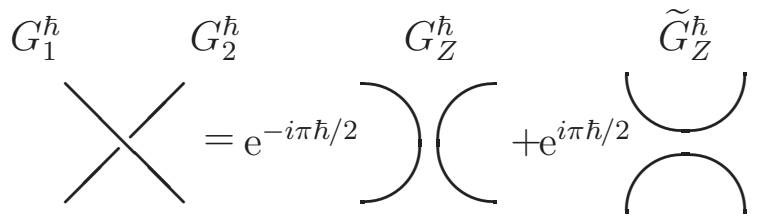

In (6.5) the order of the crossing of $G_{1}^{\hbar}$ and $G_{2}^{\hbar}$ indicates which geodesic occupies the first place in the product; the rest of the graph is unchanged).

If two QGLs have more than one intersection, the quantum skein relations must be applied simultaneously at all intersection points [15. In particular, this implies the Riedemeister moves for a graph, if we set the empty loop to be $-\mathrm{e}^{-i \pi \hbar}-\mathrm{e}^{i \pi \hbar}$.

The algebra (4.1) was quantized by the deformation quantization method in [7, 8]. By imposing the quantization conditions (6.1), we obtain the quantum $s o_{q}(m)$ algebra of [8]: $a_{i j}$ commutes with $a_{k l}$ if $i<j<k<l$ or $i<k<l<j$; the elements $a_{i j}, a_{j k}$, and $a_{i k}$ constitute the $s o_{q}(3)$ subalgebra; and if $i<k<j<l$, then $\left[a_{i j}, a_{k l}\right]=\xi\left(a_{i k} a_{j l}-a_{i l} a_{j k}\right)$, where $\xi=\mathrm{e}^{-i \pi \hbar}-\mathrm{e}^{i \pi \hbar}$.

The quantum operators $\mathcal{G}_{x_{i}, x_{j}}^{\hbar}$ are determined by equation (5.7) when the Weyl ordering is assumed, and the commutation relations between the fields appearing there are

$$
\begin{aligned}
& {\left[X^{1,2}(x), X^{1,2}(y)\right]=\pi i \hbar \epsilon(x-y),} \\
& {\left[X^{1}(x), X^{2}(y)\right]=\left[X^{1,2}(x), C(y)\right]=[C(x), C(y)]=0 .}
\end{aligned}
$$

It is straightforward to check that (again assuming $x_{1}<x_{2}<x_{3}<x_{4}$ )

$$
\left[\mathcal{G}_{x_{1}, x_{3}}^{\hbar}, \mathcal{G}_{x_{2}, x_{4}}^{\hbar}\right]=\xi\left(\mathcal{G}_{x_{1}, x_{2}}^{\hbar} \mathcal{G}_{x_{3}, x_{4}}^{\hbar}-\mathcal{G}_{x_{1}, x_{4}}^{\hbar} \mathcal{G}_{x_{2}, x_{3}}^{\hbar}\right),
$$

where, as previously, the quantities in the products in the r.h.s. commute. 


\section{Conclusions}

This paper should be considered as a first step in exploring the possibilities of using infinite/continuous genus limits of Riemann surfaces in 3D gravity to describe the effects of $\mathrm{CFT} /$ string theory. For example, one can interpret the continuous-genus limit classically as describing surfaces similar to two spherelike regions (the variables $P^{1,2}(\xi)$ ) connected by infinitely many handles (the variables $C(\xi))$. It would be interesting to find an analogous treatment of the more complicated algebras of $S L(n, \mathbb{R})$ type (see [17]) for which similar constructions may have some meaning.

An interesting question is to find eigenfunctions for the quantum geodesic operators. The spectrum is obviously continuous and positive definite, and finding possible coherent states for operators (5.7) in terms of string variables seems to be interesting problem with possible applications in conformal field theory. We expect that the fermionization technique can be useful. This work is in progress. Also, worth noting is the doubling of variables (we have two sets, $X^{1}(\xi)$ and $X^{2}(\xi)$ of them). Note that the algebra (6.7) holds only when we have one or two sets of variables; adding extra (string) variables destroys this structure.

\section{Acknowledgements}

This work was supported in part by RFBR Grant No. 05-01-00498, Support Grant for Scientific Schools NSh-2052.2003.1, Programme Mathematical Methods of Nonlinear Dynamics, Istituto Nazionale di Fisica Nucleare (INFN) of Italy Iniziativa Specifica FI41, and the Italian Ministero dell' Università e della Ricerca Scientifica e Tecnologica (MIUR) under contract PRIN-2003023852 "Physics of Fundamental Interactions: gauge theories, gravity and strings".

\section{References}

[1] Verlinde E.P. and Verlinde H.L., "Conformal Field Theory and Geometric Quantization", in Superstrings '89 (Trieste 1989) World Scientific Publishing, River Edge, NJ, 1990, pp.422-449.

[2] Chekhov L.O. and Fock V.V., "Quantum Teichmüller Space", Theor. Math. Phys. 120 (1999) 1245-1259. 
[3] Chekhov L.O. and Fock V.V., "Quantum Mapping Class Group, Pentagon Relation, and Geodesics", Proc. Steklov Math. Inst. 226 (1999) 149-163.

[4] Penner R.C., "The decorated Teichmüller space of punctured surfaces", Commun. Math. Phys. 113 (1987) 299-339.

[5] Fock V.V., "Combinatorial description of the moduli space of projective structures", hepth/9312193.

[6] Goldman W.M.: "Invariant functions on Lie groups and Hamiltonian flows of surface group representation", Invent. Math. 85 (1986) 263-302.

[7] Nelson J.E. and Regge T., "Homotopy Groups and $2+1$ dimensional Quantum Gravity", Nucl. Phys. B328 (1989) 190-202; Nelson J.E., Regge T. and Zertuche F., "Homotopy Groups and $2+1$ dimensional Quantum De Sitter Gravity,", Nucl. Phys. B339 (1990) 516-532.

[8] Nelson J.E. and Regge T., "2+1 Quantum Gravity", Phys. Lett. B272 (1991) 213-216; "Invariants of 2+1 Gravity", Commun. Math. Phys. 155 (1993) 561-568.

[9] Nelson J.E. and Regge T., "2 + 1 Quantum Gravity for High Genus", Class. Qu. Grav. 9 (1992) S187-S196.

[10] Havliček M., Klimyk A.V. and Pošta S., "Representations of the cyclically symmetric q-deformed algebra so[sub q](3)", J. Math. Phys. 40 (1999) 2135; Fairlie D.B., "Quantum Deformations of SU(2)", J.Phys. A23 (1990) L183.

[11] Ugaglia M., "On a Poisson structure on the space of Stokes matrices", Int. Math. Res. Not. 1999, No. 9, (1999) 473.

[12] Bondal A., "A symplectic groupoid of triangular bilinear forms and the braid groups", preprint IHES/M/00/02.

[13] Faddeev L.D., "Discrete Heisenberg-Weyl Group and Modular Group", Lett. Math. Phys. 34 (1995) 249-254.

[14] Kashaev R.M., "Quantization of Teichmüller Spaces and the Quantum Dilogarithm”, Lett. Math. Phys. 43 (1998) 105-115. 
[15] Chekhov L.O. and Fock V.V., "Observables in 3D Gravity and Geodesic Algebras", Czechoslovak J. Phys. 50 (2000) 1201-1208.

[16] Turaev V.G., "Skein quantization of Poisson algebras of loops on surfaces", Ann. Sci. Éc. Norm. Sup., Ser. 424 (1991) 635-704.

[17] Fock V.V. and Goncharov A.B., "Cluster ensembles, quantization and the dilogarithm", math.AG/0311245. 